\title{
LOCALLY $P$-CLOSED SPACES AND RIM $P$-CLOSED SPACES
}

\author{
DIX H. PETTEY ${ }^{\prime}$
}

\begin{abstract}
It is shown in this paper that for $P=T_{2}$ or $P=T_{3}$ every locally $P$-closed space is a retract of a rim $P$-closed. locally $P$-closed space by an open. perfect retraction map. From this it follows that there exist noncompact, rim $/ /$-closed. $H$-closed spaces and noncompact, $\operatorname{rim} R$-closed, $R$-closed spaces.
\end{abstract}

1. Introduction. Recall that a $P$ space ( $P$ a topological property) is said to be $P$-closed if it is a closed subspace of every $P$ space in which it is embedded. (Following the usual convention, we shall write $H$-closed instead of $T_{2}$-closed and $R$-closed rather that $T_{3}$-closed.) A $P$ space is defined to be locally $P$-closed (rim $P$-closed, resp.) if it has a base of open sets with $P$-closed closures ( $P$-closed boundaries, resp.).

Locally $H$-closed spaces have been studied by Obreanu [5] and Porter [6].

In a recent paper [2], Dow and Porter considered locally $R$-closed spaces and rim $R$-closed spaces. They showed that every rim $R$-closed, $R$-closed space is both locally $R$-closed and strongly minimal regular (in essentially the same way, it can be shown that every rim $H$-closed, $H$-closed space is minimal Hausdorff), and they asked if there exists a noncompact, $\operatorname{rim} R$-closed, $R$-closed space.

The main result of this paper is that for $P=T_{2}$ or $P=T_{3}$, every locally $P$-closed space is a retract of rim $P$-closed, locally $P$-closed space by an open, perfect retraction map. From this it follows that every $H$-closed space is a retract of a rim $H$-closed, $H$-closed space and that every locally $R$-closed, $R$-closed space is a retract of a rim $R$-closed, $R$-closed space. These results imply the existence of noncompact, rim $H$-closed, $H$-closed spaces and noncompact, $\operatorname{rim} R$-closed, $R$-closed spaces, thus answering the question in [2] cited above.

Remark. The definition of "locally $R$-closed" used by Dow and Porter differs slightly from the one used in this paper. They required only that each point have a base of $R$-closed neighborhoods. Their proof that a rim $R$-closed, $R$-closed space is locally $R$-closed is, however, adequate under either definition.

2. Preliminaries. Most of the results presented in this section will be used in $\S 3$. Except for Lemma 2.8 they are explicitly concerned with $P$-closed spaces, locally $P$-closed spaces, or rim $P$-closed spaces.

By a map we always mean a continuous function.

Received by the editors October 26, 1981 and, in revised form, March 10, 1982.

1980 Mathematics Subject Classification. Primary 54D25; Secondary 54C15.

$K e y$ words and phrases. $P$-closed space, locally $P$-closed space, rim $P$-closed space.

${ }^{1}$ This research was funded in part by a grant from the Research Council of the Graduate School of the University of Missouri at Columbia.

(1983 American Mathematical Society $0002-9939 / 82 / 0000-0589 / \$ 02.25$ 
2.1. Proposition. If $P=T_{2}$ or $P=T_{3}, X$ is a locally $P$-closed space, and $Y$ is a $P$ space that is the image of $X$ under an open map, then $Y$ is locally P-closed.

Proof. It is well known that for $P=T_{2}$ or $P=T_{3}$ the $P$-closed property is preserved by maps onto $P$-spaces (see [1, Theorems 3.3(b) and 4.16(b)]). In light of this, the argument is routine and straightforward (cf. [3, Theorem 3.3.15]).

The next result is not used elsewhere in the paper but is easily proved, and it seems appropriate to include it here.

2.2. Proposition. If $X$ is a locally $H$-closed space and $Y$ is the image of $X$ under a perfect map then $Y$ is locally $H$-closed.

Proof. It follows from [3, Theorem 3.7.20] that $Y$ is a $T_{2}$ space. The rest of the proof is similar to that of [3, Theorem 3.7.21]. Let $y$ be a point of $Y$ and $G$ a neighborhood of $y$, and let $f$ be a perfect map from $X$ onto $Y$. Then $f^{-1}(y)$ is compact, so there is an open neighborhood $V$ of $f^{-1}(y)$ such that $V \subseteq f^{-1}(G)$ and $\operatorname{cl} V$ is $H$-closed. Let $U=Y-f(X-V)$. Since $f$ is a closed map, $U$ is an open neighborhood of $y$. Furthermore, $U \subseteq G$. We also have $U \subseteq f(\operatorname{cl} V)$, and since $f(\mathrm{cl} V)$ is $H$-closed (see [1, Theorems 3.3(b)]) it follows from [1, Theorems 3.3(d)] that $\operatorname{cl} U$ is $H$-closed.

2.3. Proposition. If $P=T_{2}$ or $P=T_{3}, X$ is a $P$ space, $Y$ is a $P$-closed space, and there is an open, perfect map from $X$ onto $Y$, then $X$ is $P$-closed.

(This follows immediately from [4, Theorem 2.7$]$.)

2.4. Lemma. Suppose $P=T_{2}$ or $P=T_{3}$, that $X$ and $Y$ are $P$ spaces, and that $f$ is an open, perfect map from $X$ onto $Y$. If $V$ is an open subset of $Y$ and $\operatorname{cl} V$ (bd $V$, resp.) is $P$-closed, then $\mathrm{cl} f^{-1}(V)$ (bd $f^{-1}(V)$, resp.) is $P$-closed.

Proof. Since $f$ is open, we have $\mathrm{cl}^{-1}(V)=f^{-1}(\mathrm{cl} V)$ and bd $f^{-1}(V)=f^{-1}(\mathrm{bd} V)$. In light of Proposition 2.3, this completes the proof.

We use the notation $\left\langle Y_{\alpha} ; f_{\gamma}^{\alpha} ; \mathcal{D}\right\rangle$ to indicate an inverse system of topological spaces and bonding maps indexed over a directed set $\mathcal{O D}$, and we write inv $\lim _{x \in \mathcal{D}} Y_{\alpha}$ to denote the inverse limit space associated with the system. It will always be understood that for each $\alpha$ in $\left.{ }_{0}\right)$ the bonding map $f_{\alpha}^{\alpha}$ from $Y_{\alpha}$ to $Y_{\alpha}$ is the identity map. For a discussion of basic properties of inverse systems and inverse limits, see [3, pp. 135-144] (or any of several other standard topology reference works).

2.5. Proposition. If $P=T_{2}$ or $P=T_{3}$ and if $\left\langle Y_{\alpha} ; f_{\gamma}^{\alpha}\right.$; OQ $\rangle$ is an inverse system of locally $P$-closed (rim P-closed, resp.) spaces and open, perfect, onto bounding maps, then inv $\lim _{\alpha \in \vee D} Y_{\alpha}$ is locally P-closed (rim P-closed, resp.) and for each $\eta$ in of the projection from inv $\lim _{\alpha \in \mathcal{D}} Y_{\alpha}$ to $Y_{\eta}$ is an open, perfect, onto map.

Proof. It follows from [3, Theorem 2.5.2] that the inverse limit space is a $P$ space and from [4, Lemmas 2.5 and 2.4] that each projection is an open, perfect, onto map. Therefore, [3, Proposition 2.5.5] and Proposition 2.3 imply that inv $\lim _{\alpha \in \text { oI }} Y_{\alpha}$ is locally $P$-closed (rim $P$-closed, resp.). 
2.6. Proposition. If $P=T_{2}$ and $P=T_{3}$ and if $S$ is a locally $P$-closed (rim $P$-closed, resp.) space and $K$ is a compact $T_{2}$ space, then $S \times K$ is locally P-closed (rim P-closed, resp.).

(This follows by straightforward argument as a consequence of [1, Theorems 3.3(c) and 4.17(b)].)

Given a topological space $Y$, we let $w(Y)$ denote the weight of $Y$ (i.e. the smallest cardinal $\kappa$ such that $Y$ has a base of cardinality $\kappa$ ).

2.7. Lemma. If $Y$ is a locally $P$-closed space then there is a base $\mathscr{B}$ for $Y$ such that the cardinality of $\mathscr{B}$ is $w(Y)$ and such that each member of $\mathscr{B}$ has $P$-closed closure.

(This follows immediately from [3, Theorem 1.1.15].)

For each ordinal $\alpha$, we let $W(\alpha)$ denote the set of all ordinals less than $\alpha$.

2.8. Lemma. Let $\kappa$ be a cardinal number and $\beta$ an ordinal of cardinality less than or equal to $\kappa$. If $\left\langle Y_{\alpha} ; f_{\gamma}^{\alpha} ; W(\beta)\right\rangle$ is an inverse system of $T_{2}$ spaces and perfect, onto bonding maps and if $w\left(Y_{\alpha}\right)=\kappa$ for each $\alpha$ in $W(\beta)$, then the weight of inv $\lim _{\alpha<\beta} Y_{\alpha}$ is $\kappa$.

(This is an immediate consequence of [4, Lemma 2.4, 3, 3.7.19 and 4, Lemma 2.2].)

3. Retracts of rim $P$-closed spaces. In this section, we show that if $P=T_{2}$ or $P=T_{3}$ then every locally $P$-closed space is a retract of a rim $P$-closed, locally $P$-closed space by an open, perfect retraction map. It then follows that every $H$-closed space is a retract of a rim $H$-closed, $H$-closed space and that every locally $R$-closed, $R$-closed space is a retract of a rim $R$-closed, $R$-closed space.

Let $I$ denote the closed unit interval on the real line.

For a topological space $S$ and an open subset $V$ of $S$, let $Z(S, V)$ denote the quotient space obtained from $S \times I$ by identifying $(s, t)$ with $(s, 0)$ for each point $s$ of $S-V$ and each $t$ in $I$.

3.1. Lemma. If $S$ is a topological space and $V$ is an open subset of $S$, then the quotient map from $S \times I$ onto $Z(S, V)$ is perfect.

Proof. It is easily verified that the decomposition of $S \times I$ associated with the map is upper-semicontinuous and thus that the map is closed. Since point inverses are compact, this completes the proof.

3.2. Lemma. Suppose $P=T_{2}$ or $P=T_{3}$, and let $S$ be. a $P$ space and $V$ an open subset of $S$. There is a subspace $A$ of $Z(S, V)$ and an open, perfect map $\phi$ from $Z(S, V)$ onto $S$ such that

(1) $\phi \mid A$ is a homeomorphism onto $S$ and

(2) if $\operatorname{cl} V$ is $P$-closed then $\phi^{-1}(V)$ is the union of two open sets having P-closed boundaries.

Proof. Let $\mu$ denote the quotient map from $S \times I$ onto $Z(S, V)$ and $\pi$ the projection from $S \times I$ onto $S$. Let $\phi=\pi \mu^{-1}$. It is easily verified that $\phi$ is a well-defined function from $Z(S, V)$ onto $S$. Since $\phi^{-1}=\mu \pi^{-1}$ and $\mu$ is closed 
(Lemma 3.1), $\phi$ is continuous. Since the map $\pi$ is open and perfect, it follows that $\phi$ is also. Let $A$ denote the subspace $\mu(S \times\{0\})$ of $Z(S, V)$. Then $\phi \mid A$ is a homeomorphism onto $S$. Suppose now that $\mathrm{cl} V$ is $P$-closed, and observe that $\phi^{-1}(V)$ is the union of the two open subsets $\mu(V \times[I-\{0\}])$ and $\mu(V \times[I-\{1\}])$ of $Z(S, V)$. It is not difficult to see that bd $\mu(V \times[I-\{0\}])=\mu([\operatorname{cl} V] \times\{0\})$ and that bd $\mu(V \times[I-\{1\}])=\mu([\mathrm{cl} V] \times\{1\})$. Since $\mathrm{cl} V$ is $P$-closed, we conclude that each of bd $\mu(V \times[I-\{0\}])$ and bd $\mu(V \times[I-\{1\}])$ is $P$-closed.

3.3. Lemma. If $P=T_{2}$ or $P=T_{3}$ and if $S$ is a locally $P$-closed space of infinite weight and $V$ is an open subset of $S$, then $Z(S, V)$ is a locally P-closed space of the same weight as $S$.

Proof. Since $S$ is a locally $P$-closed space, it follows that $S \times I$ is a locally $P$-closed space (Proposition 2.6) and thus that $Z(S, V)$ is a $P$ space (Lemma 3.1 and [3, Theorem 3.7.20]). To show $Z(S, V)$ is locally $P$-closed, let $z$ be a point of $Z(S, V)$ and $N$ an open neighborhood of $z$. Let $\mu, \pi$, and $\phi$ be as in the proof of Lemma 3.2. Consider first the case where $\phi(z) \in V$. Then $\mu^{-1}(z)$ is a singleton subset of $V \times I$, and we can choose an open subset $U$ of $S \times I$ such that $\mu^{-1}(z) \subseteq U \subseteq(V \times I) \cap$ $\mu^{-1}(N)$ and cl $U$ is $P$-closed. Further, $z \in \mu(U) \subseteq N$. Because $\mu$ takes $V \times I$ homeomorphically onto an open subset of $Z(S, V), \mu(U)$ is an open neighborhood of $z$. Since $\mu$ is perfect we have $\operatorname{cl} \mu(U)=\mu(\operatorname{cl} U)$, implying that $\operatorname{cl} \mu(U)$ is $P$-closed. Consider now the case where $\phi(z) \in S-V$. Then $\mu^{-1}(z)=\{\phi(z)\} \times I$, and there thus exists an open subset $G$ of $S$ such that $\mu^{-1}(z) \subseteq G \times I \subseteq \mu^{-1}(N)$ and $\operatorname{cl} G$ is $P$-closed. Then $z \in \phi^{-1}(G)=\mu(G \times I) \subseteq N$. Since $\phi$ is continucus, open, and perfect it follows that $\phi^{-1}(G)$ is open and that $\mathrm{cl}^{-1}(G)$ is $P$-closed (Lemma 2.4). Hence, $Z(S, V)$ is locally $P$-closed. Finally, observe that $w(S)$ being infinite implies that $w(S)=w(S \times I)$. Perfect maps cannot increase weight [3, Theorem 3.7.19], so Lemmas 3.1 implies that $w(Z(S, V)) \leqslant w(S \times I)$ and Lemma 3.2 implies that $w(S) \leqslant w(Z(S, V))$. Therefore, $w(Z(S, V))=w(S)$.

In proving the next theorem, we use a modification of a technique used by Louis Friedler and this author in the proof of [4, Theorem 2.8]. (Cf. also [7, §3].)

3.4. TheOREM. If $P=T_{2}$ or $P=T_{3}$ and if $Y$ is a locally $P$-closed space, then there is a rim $P$-closed, locally $P$-closed space $X$ of the same weight as $Y$ such that

(1) $Y$ is a subspace of $X$ and

(2) there is an open, perfect retraction from $X$ onto $Y$.

Proof. We may assume $w(Y)$ to be infinite (for otherwise $Y$ has only finitely many points and is thus compact). Let $\tau$ denote the least ordinal of cardinality $w(Y)$, and let $\sigma$ denote a one-to-one function from $W(\tau) \times W(\tau)$ onto $W(\tau)-\{0\}$ such that $\sigma(\alpha, \zeta)>\alpha$ for each $(\alpha, \zeta)$ in $W(\tau) \times W(\tau)[4$, Lemma 2.1].

We choose by transfinite induction, in the manner indicated below, an inverse system $\left\langle Y_{\alpha} ; f_{\gamma}^{\alpha} ; W(\tau)\right\rangle$ of locally $P$-closed spaces and open, perfect, onto bonding maps such that for each $\alpha$ in $W(\tau), w\left(Y_{\alpha}\right)=w(Y)$. For each $\alpha$ we also choose a base $\mathscr{G}_{\alpha}=\left\{B_{\alpha \zeta} \mid \zeta<\tau\right\}$ for $Y_{\beta}$ such that each member of ${ }_{i h}$ has a $P$-closed closure. The spaces, bonding maps, and bases are chosen according to the following procedure. 
Let $Y_{0}=Y$, and let $f_{0}^{0}$ be the identity map on $Y_{0}$. Choose $\cup_{0}=\left\{B_{0 \zeta} \mid \zeta<\tau\right\}$ to be a base for $Y_{0}$ such that each member of ${ }^{\prime} B_{0}$ has $P$-closed closure (see Lemma 2.7).

Suppose now that $\beta$ is a nonzero ordinal in $W(\tau)$, that the inverse system $\left\langle Y_{\alpha} ; f_{\gamma}^{\alpha} ; W(\beta)\right\rangle$ of locally $P$-closed spaces and open, perfect, onto bonding maps has already been chosen, and that for each $\alpha$ in $W(\beta)$ the base ${ }_{0} \beta_{\alpha}=\left\{B_{\alpha \zeta} \mid \zeta<\tau\right\}$ has been chosen (with each member of $G_{h}$ having $P$-closed closure). Let $S_{\beta}=$ inv $\lim _{\alpha<\beta} Y_{\alpha}$, and for each $\alpha$ in $W(\beta)$ let $g_{\alpha}^{\beta}$ denote the open, perfect projection from $S_{\beta}$ onto $Y_{\alpha}$ (see Proposition 2.5). Then $S_{\beta}$ is a locally $P$-closed space, and $w\left(S_{\beta}\right)=w(Y)$ (Proposition 2.5 and Lemma 2.8). Let $(\eta, \xi)$ be the ordered pair in $W(\tau) \times W(\tau)$ such that $\sigma(\eta, \xi)=\beta$. Then $\eta<\beta$, which implies that $Y_{\eta}$ and $(i) \eta$ have already been chosen. Let $V_{\beta}$ denote the open subset $\left(g_{\eta}^{\beta}\right)^{-1}\left(B_{\eta \xi}\right)$ of $S_{\beta}$. Then $V_{\beta}$ has $P$-closed closure (Lemma 2.4). Now let $Y_{\beta}=Z\left(S_{\beta}, V_{\beta}\right)$, and let $A_{\beta}$ be a subspace of $Y_{\beta}$ and $\phi_{\beta}$ an open, perfect map from $Y_{\beta}$ onto $S_{\beta}$ such that (1) $\phi_{\beta} \mid A_{\beta}$ is a homeomorphism onto $S_{\beta}$ and (2) $\left(\phi_{\beta}\right)^{-1}\left(V_{\beta}\right)$ is the union of two open sets having $P$-closed boundaries (see Lemma 3.2). Then $Y_{\beta}$ is a locally $P$-closed space such that $w\left(Y_{\beta}\right)=w(Y)$ (Lemma 3.3). Choose $\cup_{\beta}=\left\{B_{\beta \zeta} \mid \zeta<\tau\right\}$ to be a base for $Y_{\beta}$ such that each member of $\mathscr{G}_{\beta}$ has $P$-closed closure. Now let $f_{\beta}^{\beta}$ denote the identity map on $Y_{\beta}$, and for each $\alpha$ in $W(\beta)$ let $f_{\alpha}^{\beta}$ denote the open perfect map $g_{\alpha}^{\beta} \phi_{\beta}$ from $Y_{\beta}$ onto $Y_{\alpha}$. Then $f_{\gamma}^{\beta}=f_{\gamma}^{\alpha} f_{\alpha}^{\beta}$ whenever $\gamma \leqslant \alpha \leqslant \beta$, and $\left(\phi_{\beta}\right)^{-1}\left(V_{\beta}\right)=\left(f_{\eta}^{\beta}\right)^{-1}\left(B_{\eta \xi}\right)$.

Having inductively generated the system $\left\langle Y_{\alpha} ; f_{\gamma}^{\alpha} ; W(\tau)\right\rangle$ and the corresponding bases $\mathscr{B}_{\alpha}(\alpha<\tau)$, we now let $X=$ inv $\lim _{\alpha<\tau} Y_{\alpha}$, and for each $\alpha$ in $W(\tau)$ we let $f_{\alpha}$ denote the projection from $X$ to $Y_{\alpha}$.

It follows from Proposition 2.5 and Lemma 2.8 that $X$ is a locally $P$-closed space of weight $w(Y)$ and that each projection $f_{\alpha}(\alpha<\tau)$ is open, perfect, and onto. To show that $X$ is $\operatorname{rim} P$-closed, let $x$ be a point of $X$ and $G$ an open neighborhood of $x$. Then there is an ordered pair $(\eta, \xi)$ in $W(\tau) \times W(\tau)$ such that $x \in\left(f_{\eta}\right)^{-1}\left(B_{\eta \xi}\right) \subseteq G$ (see [3, Proposition 2.5.5]). Let $\beta$ be the ordinal $\sigma(\eta, \xi)$. Then $\left(f_{\eta}^{\beta}\right)^{-1}\left(B_{\eta \xi}\right)$ $\left(=\left(\phi_{\beta}\right)^{-1}\left(V_{\beta}\right)\right)$ is the union of two open sets having $P$-closed boundaries. Choose $U$ to be one of these two open sets and such that $f_{\beta}(x) \in U$. Then $x \in\left(f_{\beta}\right)^{-1}(U) \subseteq G$, and bd $\left(f_{\beta}\right)^{-1}(U)$ is $P$-closed (Lemma 2.4 ).

It remains to be shown that $Y$ may be embedded in $X$ in such a way that there is an open, perfect retraction from $X$ onto $Y$. To do this, it is sufficient to show the existence of a subspace $H$ of $X$ such that $f_{0} \mid H$ is a homeomorphism onto $Y_{0}$. We let $H_{0}=Y_{0}$ and choose by induction a family $\left\{H_{\alpha} \subseteq Y_{\alpha} \mid \alpha<\tau\right\}$ of subspaces such that for each $\alpha$ in $W(\tau)$ and each $\gamma$ in $W(\alpha+1)$ the restriction $f_{\gamma}^{\alpha} \mid H_{\alpha}$ is a homeomorphism onto $H_{\gamma}$. For each nonzero ordinal $\beta$ in $W(\tau)$ (assuming the collection $\left\{H_{\alpha} \mid \alpha<\beta\right\}$ has already been chosen) we let $H_{\beta}^{\prime}$ denote the subspace inv $\lim _{\alpha<\beta} H_{\alpha}$ of $S_{\beta}$ and then choose $H_{\beta}$ to be a subspace of $A_{\beta}$ such that $\phi_{\beta} \mid H_{\beta}$ is a homeomorphism onto $H_{\beta}^{\prime}$. It follows from our construction and [3, Proposition 2.5.10] that for each $\alpha$ in $W(\tau)$ and each $\gamma$ in $W(\alpha+1), f_{\gamma}^{\alpha} \mid H_{\alpha}$ is a homeomorphism onto $H_{\gamma}$. Now let $H$ denote the subspace inv $\lim _{\alpha<\tau} H_{\alpha}$ of $X$. Then $f_{0} \mid H$ is a homeomorphism onto $Y_{0}[3$, Proposition 2.5.10]. This completes the proof.

Every $H$-closed space is locally $H$-closed (see [1, Theorem 3.3(d)]). Therefore, the next result is an immediate consequence of Theorem 3.4 and Proposition 2.3. 
3.5. THEOREM. If $Y$ is an $H$-closed space, then there exists a rim $H$-closed, $H$-closed space $X$ of the same weight as $Y$ such that (1) $Y$ is a subspace of $X$ and (2) there is an open, perfect retraction from $X$ onto $Y$.

Similarly, we have the following.

3.6. THEOREM. If $Y$ is a locally $R$-closed, $R$-closed space, then there exists a rim $R$-closed, $R$-closed space $X$ of the same weight as $Y$ such that (1) $Y$ is a subspace $X$ and (2) there is an open, perfect retraction from $X$ onto $Y$.

(There exist $R$-closed spaces that are not locally $R$-closed $[2,2.7]$, so in light of Proposition 2.1 it is clear that the locally $R$-closed requirement cannot be removed from the hypothesis of Theorem 3.6.)

There are known examples of noncompact, $H$-closed spaces [1, Example 3.13] and noncompact, locally $R$-closed, $R$-closed spaces [1, Example 4.18]. Therefore, Theorems 3.5 and 3.6 immediately imply the next result. This settles Problem 4.3 of [2].

3.7. COROllary. There exist noncompact, rim H-closed, H-closed spaces and noncompact, rim $R$-closed, $R$-closed spaces.

\section{REFERENCES}

I. M. P. Berri, J. R. Porter and R. M. Stephenson, Jr., A survey of minimal topological spaces, General Topology and its Relation to Modern Analysis and Algebra. III, Proc. Kanpur Topology Conf. (1968). Academia, Prague, 1971, pp. 93-114.

2. Alan Dow and Jack Porter, Embedding in R-closed spaces, Topology Appl. (to appear).

3. Ryszard Engelking, General topologv. PWN, Warsaw, 1977.

4. Louis M. Friedler and Dix H. Pettey, Inverse limits and mappings of minimal topological spaces, Pacific J. Math. 71 (1977), 429-448.

5. F. Obreanu. Espaces localement absolument fermés, Ann. Acad. Repub. Pop. Romaine Sect. Sti. Fiz. Chim. Ser. A 3 (1950), 375-394.

6. Jack Porter, On locally H-closed spaces, Proc. London Math. Soc. (3) 20 (1970), 193-204.

7. T. O. Vinson. Jr. and R. F. Dickman, Jr., Inverse limits and absolutes of H-closed spaces. Proc. Amer. Math. Soc. 66 (1977), 351-358.

Department of Mathematics, University of Missouri, Columbia, Missouri 65211 